\title{
Flexible Pressure, Temperature, and Flow Microsensors for Integration in Methanol Microreformer
}

\author{
Chi-Yuan Lee*, Chia-Chieh Shen, Yen-Ting Cheng and Yu-Ming Chang \\ Department of Mechanical Engineering, Yuan Ze Fuel Cell Center, Yuan Ze University, \\ Taoyuan, 320, Taiwan, R.O.C.
}

(Received November 5, 2012; accepted February 4, 2013)

Key words: $\quad$ MEMS, methanol microreformer, PI, pressure, temperature, flow microsensor

In recent years, the development of fuel cells has proceeded rapidly, and so the reformation of methanol to produce hydrogen has become a serious problem. Supplying hydrogen from a methanol microreformer to fuel cells is an important topic. The structure of a microflow channel must support the transfer of external heat to the reform reaction, facilitating the diffusion of methanol vapor into the catalyst layer, thereby increasing the rate of transfer of hydrogen. In this investigation, the micro-electromechanical systems (MEMS) technique is utilized to fabricate pressure, temperature, and flow microsensors. Polyimide film (PI) exhibits high temperature resistance and stress corrosion resistance, and is adopted herein as a flexible substrate. Multifunctional microsensors are inserted into methanol microreformers to measure the internal pressure, temperature, and flow in situ, and the signals thus obtained are used to improve their efficiency.

\section{Introduction}

Energy shortages have become increasingly severe in recent years, motivating the development of green energy technologies. As an emerging green energy technology, fuel cells are replacing petrochemical fuel as a major energy source. Hydrogen vehicle technologies are being considered as possible solutions to mitigate environmental burdens and fossil fuel dependence. ${ }^{(1)}$ Proton exchange membrane fuel cells (PEMFCs) use hydrogen and oxygen as fuel. Oxygen is extracted from the atmosphere, and hydrogen can be generated from fossil fuels. The main processes involved are steam reforming, partial oxidation and coal gasification. Hydrogen can also be formed from nonfossil fuels by water splitting, photoelectrochemical water splitting ${ }^{(2,3)}$ and a few other methods. Reformers are widely utilized in fuel cells because fossil fuels are easily obtained and reforming can produce large amounts of hydrogen.

*Corresponding author: e-mail: cylee@saturn.yzu.edu.tw 
As a liquid fuel for use in a reformer, methanol is favored over hydrogen because it can be very safely stored and transported, and has a high carbon/hydrogen ratio, high power density and low reforming temperature $\left(250-300{ }^{\circ} \mathrm{C}\right) .(4)$ However, problems related to its use in various applications include the starting time and its power density. The efficient and steady production of hydrogen is another important issue.

$\mathrm{Kim}^{(5)}$ proposed a methanol microreformer that comprised a fuel vaporizer/pre-heater, a steam reformer, a combustor/heat exchanger, and a preferential oxidation (PROX) reactor. The methanol is reformed by the reforming catalyst to generate hydrogen in the steam reformer. To supply heat to the steam reformer, some of the hydrogen that is not utilized at the anode of the fuel cell is fed to the combustor to generate sufficient heat to sustain the steam reforming of the methanol. Kim achieved a conversion of methanol of $95.7 \%$ and a hydrogen flow rate of $53.7 \mathrm{ml} / \mathrm{min}$, with $1.24 \%$ carbon monoxide.

Hsueh et al. ${ }^{(6)}$ numerically studied the transport phenomena and performance of a plate methanol steam microreformer with a serpentine flow field at various wall temperatures, fuel ratios and Reynolds numbers.

Kwon et al. ${ }^{(7)}$ fabricated a microflow plate using silicon fabrication technology and a new catalyst loading method called fill-and-dry coating. The microreformer stack, which comprised one vaporizer and two reformers, occupied a volume of $15 \mathrm{~cm}^{3}$. The operating temperature of the reformer was in the range of $280-320{ }^{\circ} \mathrm{C}$. The maximum hydrogen production rate and conversion were approximately $200 \mathrm{~cm}^{3}$ and $95 \%$ at $320{ }^{\circ} \mathrm{C}$, respectively.

In the authors' other work, a flexible temperature microsensor was successfully embedded in a methanol microreformer for in situ diagnosis of inner local temperature distributions during the oxidative and steam reforming of methanol. ${ }^{(8,9)}$ In the present work, three-in-one pressure, temperature, and flow flexible microsensors are integrated on a polyimide film substrate. The multifunctional microsensors are inserted into methanol microreformers to measure the internal pressure, temperature and flow signals in situ, and these signals are used to increase the efficiency of the methanol microreformer.

\section{Methodology}

\subsection{Theory of temperature sensor}

A resistance temperature detector (RTD) was used as a temperature sensor. Since a metal conductor has a positive temperature coefficient (PTC), the resistance of an RTD increased with environmental temperature. In a situation in which the temperature variation of an RTD is linear, the relationship between the measured resistance and temperature change is given by

$$
R_{t}=R_{r}(1+\alpha \Delta T)
$$

where $R_{t}$ is the resistance at $t^{\circ} \mathrm{C}, R_{r}$ is the resistance at $r{ }^{\circ} \mathrm{C}$, and $\alpha$ is sensitivity. In the linear range, eq. (1) can be rewritten as eq. (2). The temperature can be determined from the resistance using a calibration curve.

$$
\alpha_{T}=\frac{R_{t}-R_{i}}{R_{i}(\Delta T)},
$$


Figure 1 schematically depicts the serpentine structure of an RTD temperature microsensor $\left(620 \times 600 \mu \mathrm{m}^{2}\right)$.

\subsection{Theory of pressure sensor}

Conventional pressure sensors can be classified as piezoresistive, capacitive, or resonant. ${ }^{(10-13)}$ A piezoresistive pressure sensor is extremely sensitive to temperature; therefore, it is unsuitable for use in high-temperature environments. A capacitive pressure sensor is less sensitive to temperature, but its sensitivity is higher at lower pressure. ${ }^{(14)}$ The fabrication of resonant pressure sensors is very complex. For all these reasons, the capacitive pressure sensor is the best suited for use in reformers.

Figure 2 schematically depicts a capacitive pressure sensor $\left(1200 \times 800 \mu \mathrm{m}^{2}\right)$. When pressure $P$ is applied to the electrode surface, the gap between the two parallel electrodes changes as the dielectric layer deforms, so the capacitance instantaneously changes. Equation (3) describes the operation of a capacitive pressure sensor,

$$
\Delta C=\varepsilon_{r} \varepsilon_{0} \frac{A}{\Delta d}
$$

where $\Delta C$ denotes the change in capacitance, $\varepsilon_{r}$ represents the dielectric constant, $\varepsilon_{0}$ is the dielectric constant in a vacuum, $A$ denotes the area of overlap of the two parallel electrodes, and $\Delta d$ is the change in the distance between the two parallel electrodes under an applied pressure, which is identically the change in thickness of the dielectric layer.

\subsection{Theory of flow sensor}

Flow sensors are classified into thermal flow sensors and nonthermal flow sensors. The simple design of a flow sensor makes its fabrication simple. A hot-wire flow sensor is adopted herein. The structure of a hot-wire flow sensor is basically that of a serpentine microheater. The operation of a hot-wire flow sensor involves the supply of a constant voltage to it, causing it to heat the environment, which eventually has a stable temperature. If the flow sensor is placed in a constant flow field, then its temperature changes, as displayed in Fig. 3. In a hot wire, Joule heating converts electrical energy to thermal energy. The increase in wire temperature changes the resistance to an extent that is determined by the temperature coefficient of resistance of the material of the wire. According to ref. 15, eq. (4) gives the relationship between the rate of thermal energy dissipation and the flow speed.
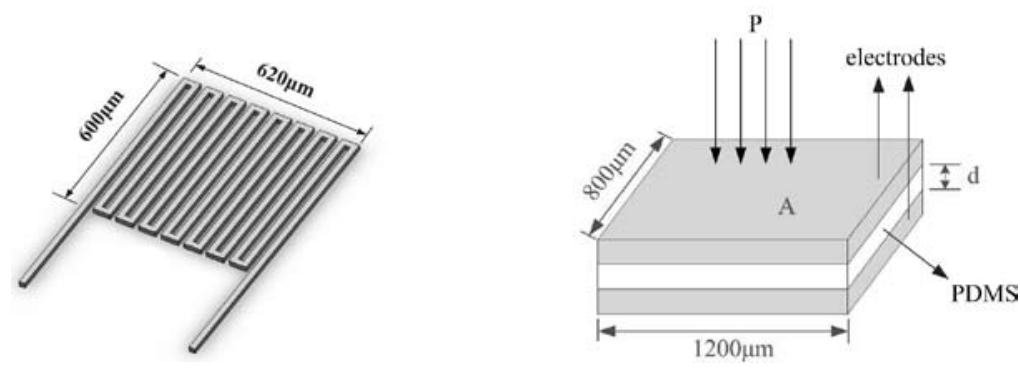

Fig. 1 (left). RTD temperature sensor.

Fig. 2 (right). Capacitive pressure sensor. 


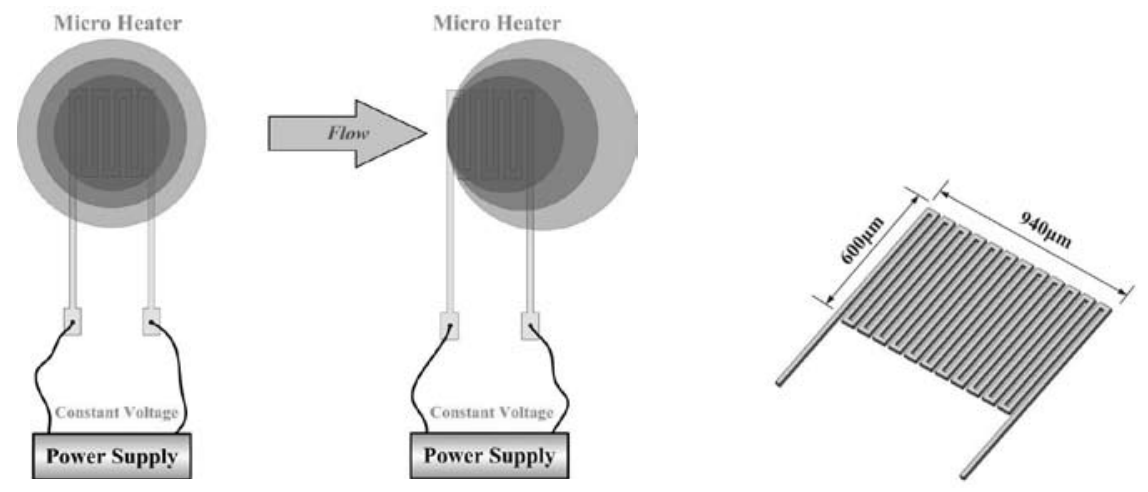

Fig. 3 (left). Theory of flow sensor.

Fig. 4 (right). Schematic diagram of flow sensor.

$$
Q=I^{2} \times R=I \times V=\left(A+B \times U^{n}\right)\left(T_{\mathrm{s}}-T_{\mathrm{o}}\right),
$$

where $Q$ represents the power that is supplied by the external power supply to a hot wire, $U$ denotes the flow speed, $T_{\mathrm{s}}$ represents the temperature of the hot wire, $T_{\mathrm{o}}$ is the flow temperature, and $n$ is the coefficient that relates $U$ to $Q$. $A$ is a constant coefficient of the transfer of energy by the heater at a flow speed of zero, whereas $B$ is a constant coefficient of the transfer of energy by the heater at a nonzero flow speed.

A temperature microsensor can be also adopted as a flow microsensor. ${ }^{(16)}$ Figure 4 schematically depicts a flow microsensor $\left(940 \times 600 \mu \mathrm{m}^{2}\right)$.

\section{Fabrication of Flexible Microsensors}

Figure 5 presents the fabrication processes of flexible microsensors. They are as follows. (a) Glass was used as the upholder. (b) Polyimide film is known for thermal stability, good chemical resistance, and excellent mechanical properties. A polyimide film was utilized as the flexible substrate and insulation layer. The particles on the polyimide film were removed using acetone and methanol. (c) An E-beam evaporator was used to evaporate chromium ( $\mathrm{Cr}$ ) as the adhesion layer and then gold ( $\mathrm{Au}$ ) on the chromium film as the sensing layer. Microsensors were patterned by a lithographic process and wet etching. (d) Copper was evaporated on the microsensors and the pad as a hard mask. (e) Sylgard 184 polydimethylsiloxane (PDMS) from DOW CORNING ${ }^{\circledR}$ was mixed with a base and a curing agent in an appropriate ratio, and then a vacuum chamber was degassed. The PDMS mixture solvent was then spin-coated onto the polyimide film. (f) The PDMS mixture solvent was then cured in an oven. The PDMS surface was hydrophobic, and plasma oxidation treatment made it hydrophilic. (g) Copper was deposited for a second time as a hard mask to prevent the etching away of PDMS by reactive ion etching (RIE). (h) PDMS was patterned by RIE. (i) Copper was removed using a $\mathrm{FeCl}_{3}$ etching solvent and the PDMS surface was again treated with plasma. (j) Copper was patterned as the upper electrode of a capacitive pressure microsensor. (k) A photoresist (PR) was coated on the polyimide film as a sputtering mask, and PR was patterned in the required shape. (l) Aluminum nitride (AIN) was sputtered as a top insulation layer by radio frequency (RF) sputtering. (m) Finally, AlN was etched using phosphoric acid. Figure 6 shows photographs of flexible microsensors. 


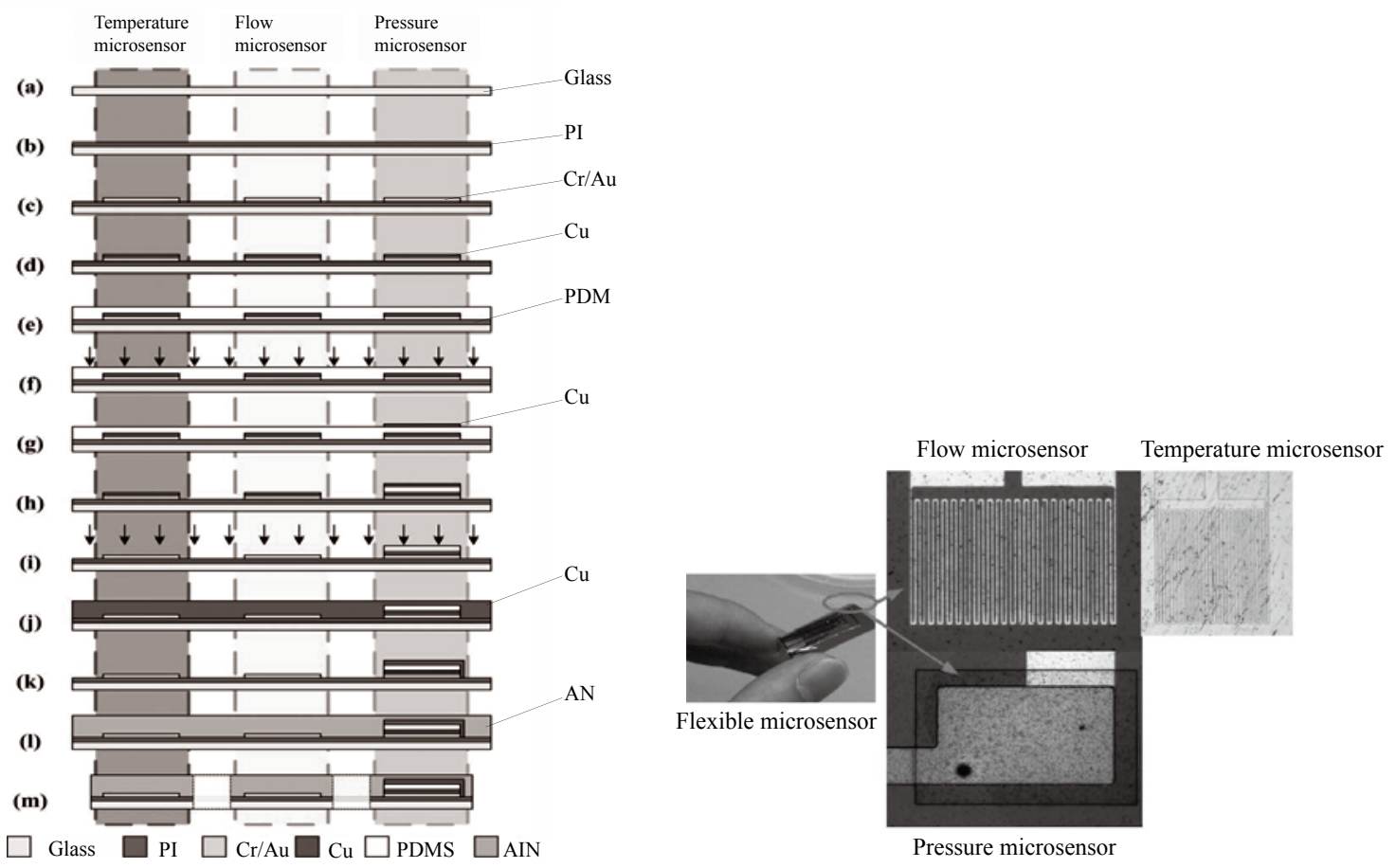

Fig. 5 (left). Fabricating flexible micro sensors on polyimide film substrate.

Fig. 6 (right). Optical microscopy image of flexible microsensors.

\section{Results and Discussion}

The inner resistance of a temperature sensor can be determined from the equation that relates temperature to resistance, and then calculating the temperature within the reformer by interpolation.

Figure 7 displays the calibration system. The sensor is firstly fabricated on the flow plate; it is then placed in the drying oven to control the temperature. An NI PXI 1033 unit is used to measure the resistance of the sensor, which can be calibrated. The temperature is calibrated from 200 to $300{ }^{\circ} \mathrm{C}$. Figure 8 plots the calibration curve, revealing that the temperature and resistance are linearly dependent on each other.

\section{Conclusions}

This work demonstrates a flexible, integrated, three-in-one pressure, temperature, and flow microsensor on a polyimide film substrate. Moreover, the temperature calibration curve reveals that the temperature and resistance are linearly dependent on each other. In future work, such microsensors will be embedded in a methanol microreformer for in situ diagnosis. They were successfully used to measure the conditions inside a reformer, providing further insight into the inner pressure, temperature and flow distributions in the steam reforming reaction of methanol. A future study will evaluate the operating parameters of flexible pressure, temperature, and flow microsensors in a microreformer with a view to improving the design and performance of microreformers. 

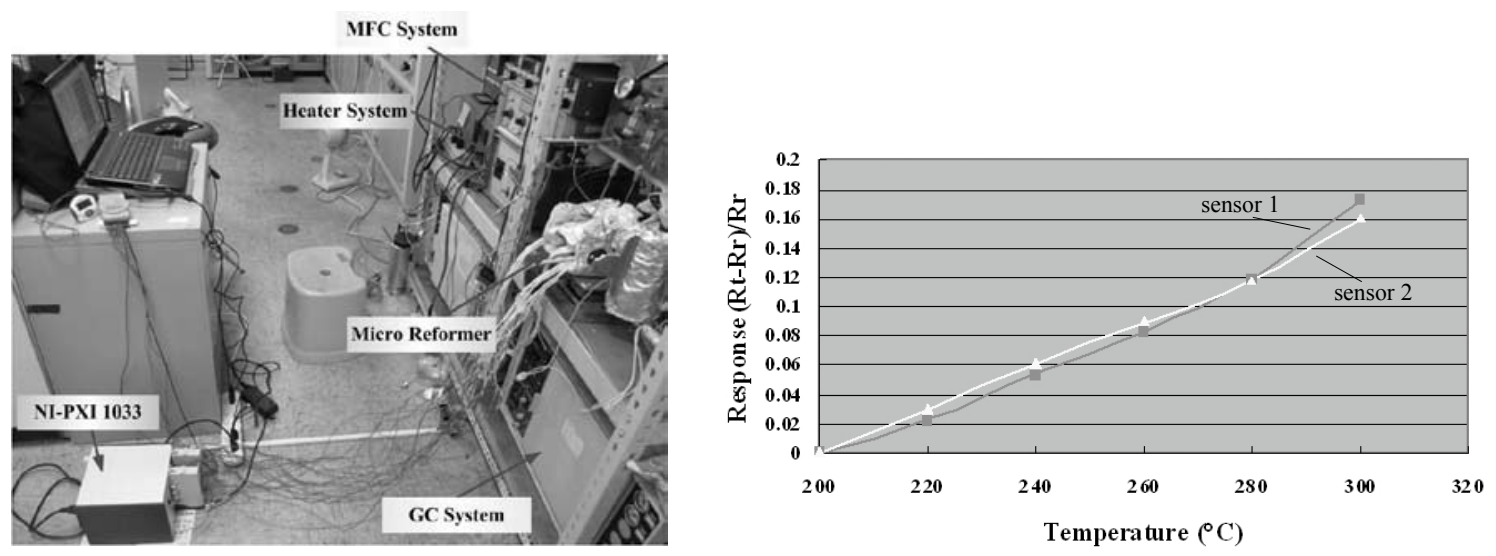

Fig. 7 (left). Calibration system.

Fig. 8 (right). Calibration curves of temperature microsensors.

\section{Acknowledgements}

This work was accomplished with much needed support, and the authors would like to thank the financial support of this research from the National Science Council of R.O.C. through grant NSC 100-2221-E-155-039-MY2, NSC 100-2221-E-155-034-MY2, and NSC 102-2622-E-155-003. The authors also like to thank Professors Chuin-Tih Yeh, Shuo-Jen Lee, Ho-Shing Wu, Kuen-Song Lin, and Yu-Chuan Lin of Yuan Ze University for their valuable advice and assistance in the experiments. In addition, we would like to thank the NENS Common Lab for providing access to their research facilities.

\section{References}

1 A. A. Boretti: Int. J. Eng. Technol. Innovation 2 (2012) 13.

2 L. J. Minggu, W. R. W. Daud and M. B. Kassim: Int. J. Hydrogen Energy 35 (2010) 5233.

3 N. A. Kelly and T. L. Gibson: Int. J. Hydrogen Energy 33 (2008) 6420.

4 C. Brunetto, A. Moschetto and G. Tina: Electr. Power Syst. Res. 79 (2009) 17.

5 T. Kim: Int. J. Hydrogen Energy 34 (2009) 6790.

6 C. Y. Hsueh, H. S. Chu, W. M. Yan and C. H. Chen: Appl. Energy 87 (2010) 3137.

7 O. J. Kwon, S. M. Hwang, J. G. Ahn and J. J. Kim: J. Power Sources. 156 (2006) 253.

8 C. Y. Lee, S. J. Lee, C. C. Shen, C. T. Yeh, C. C. Chang and Y. M. Chang: Int. J. Hydrogen Energy 36 (2010) 2869.

9 C. Y. Lee, S. J. Lee, C. C. Shen, C. T. Yeh, C. C. Chang and Y. M. Lo: Sensors 11 (2011) 2246.

10 C. C. Chiang, C. K. Lin and M. S. Ju: Sens. Actuators A 134 (2007) 382.

11 D. W. Lee and Y. S. Choi: Microelectron. Eng. 85 (2008) 1054.

12 G. Koley, J. Liu, M. W. Nomani, M. Yim, X. Wen and T. Y. Hsia: Mater. Sci. Eng. C 29 (2009) 685.

13 J. Makal: J. Vibroeng. 11 (2009) 729.

14 M. Elwenspoek and R. Wiegerink: Mechanical Microsensors (Springer, New York, 2001).

15 L. V. King: Proc. R. Soc. London A 90 (1914) 563.

16 C. Y. Lee, P. C. Chan and C. J. Lee: Sensors 10 (2010) 11605. 